\title{
Modeling and simulation in tissue biomechanics: Modern tools to face an ancient challenge
}

\author{
Giuseppe Vairo
}

Department of Civil Engineering and Computer Science (DICII), University of Rome "Tor Vergata”, Rome, Italy

Email: vairo@ing.uniroma2.it

Received 1 November 2013; revised 1 December 2013; accepted 16 December 2013

Copyright (C) 2013 Giuseppe Vairo. This is an open access article distributed under the Creative Commons Attribution License, which permits unrestricted use, distribution, and reproduction in any medium, provided the original work is properly cited.

Keywords: History of Biomechanics; Soft Tissue Biomechanics; Multiscale Homogenization Approaches; Collagen Bio-Structures

Coinage of the word "Biomechanics" is almost recent. The first to use this word was Nikolai Bernstein (18961966) by composing the Ancient Greek words Bíos (bios, that is life) and $\mu \eta \chi \alpha v i \kappa \eta$ (mēchanike, that is mechanics), aiming to address the study of the mechanical principles that regulate living organisms and biological structures. But origins of the Biomechanics can be retained really antique.

The Edwin Smith surgical papyrus, an Egyptian document written in the 17th century $\mathrm{BC}$, described the difference among cervical sprain, fracture, and fracturedislocation. By the time of Hippocrates (4th century BC), physical means such as traction or local pressure were being used to correct spinal deformities, as preliminary clinical treatments based on the first traces of spinal biomechanics [1]. Aristotle (4th century BC), starting from the deductive reasoning and the mathematical reasoning identified by Socrates and Plato as fundamental scientific tools, wrote De Motu Animalium (namely, On the Movement of Animals), wherein he described animals' bodies as complex mechanical systems. With the fall of Greece and the rise of the Roman Empire, natural philosophy waned in favor of technology. The second century anatomist, Galen, physician to the Roman emperor Marcus Aurelius, wrote a monumental work, On the Function of the Parts (referring to the parts of the human body). Perhaps, it can be considered as the prodrome of a textbook on Biomechanics. Nevertheless, the Renaissance produced the first serious attempts at formally and systematically describing biomechanical concepts, as well as at using physical laws and/or materials sciences for understanding the mechanics of many biological systems.

Leonardo da Vinci (1452-1519) studied anatomy in the context of mechanics, analyzed muscle forces as acting along lines connecting origins and insertions, investigated joint function, as well as addressed spine stability. Galileo Galilei (1564-1642) was particularly attracted by bone mechanics and basic principles of allometry. He observed that animals' masses increased disproportionately to their size, and their bones must consequently also disproportionately increase in girth, adapting to load-bearing rather than mere size. Moreover, he sought to formulate physical laws by mathematical approaches, further freeing scientific conclusions from the misperceptions of the senses. The first comprehensive treatise on Biomechanics, De Motu Animalium, was written by Giovanni Alfonso Borelli (1608-1679). It contained the first analysis of weight bearing by the spine, as well as the first explanation that the levers of the musculoskeletal system magnify motion rather than force, so that muscles must produce much larger forces than those resisting the motion. Borrelli, Marcello Malpighi (1628-1694, the much younger chair of theoretical medicine) and René Descartes (1596-1650, mathematician and father of modern Philosophy) were key thinkers in establishing the iatrophysical approach to medicine, which held that mechanics rather than chemistry was the key to understanding the functioning of the human body [2].

After Borelli, there is little sign of biomechanics in the literature until the latter half of the 19th century. The idea of investigating locomotion using cinematography was first used scientifically by Etienne Marey (1830-1904), who first correlated ground reaction forces with movement, opening to the modern motion analysis. In Germany, the Weber brothers studied human gait and computed first body segment parameters for human motion analysis (1836). Their results were later extended by Christian Wilhelm Braune and his student Otto Fischer (1889). Relationships between muscle forces and velocity of contraction were introduced and deepened by Archibald Vivian Hill (1886-1977), by using a consistent thermodynamic approach. And starting from the stress 
theory of Augustin Cauchy (1789-1857), the railroad engineer Karl Culmann (1821-1881) and the anatomist Hermann von Meyer (1801-1869) compared the stress patterns in a human femur with those in a similarly shaped crane. Their evidence led Julius Wolff (18361908) to introduce a law of bone remodeling, opening the door to the modern orthopedic medicine.

These are the origins. This is a brief and incomplete "genealogy" of Biomechanics, that clearly highlights as deep roots of Mechanics, Mathematics, Philosophy, Medicine have nourished the big tree of Biomechanics. But, what is Biomechanics today? And what is the future trend of Biomechanics?

Biomechanics is surely still the Science that aims to understand via physical laws and analytical mechanics many complex phenomena underlying living structures. It is the Science that attempts to identify factors and multi-field interactions affecting and/or activating specific biological processes. But today, in the modern insilico era, Biomechanics is also the way for trying to trace more effective diagnoses, to find solutions and therapies, and to give clinical answers against diseases and pathological processes. Biomechanics is also the set of skills and knowledge that allows to design smart medical devices and materials; that allows to face tissue engineering with the aim of repairing and restoring living tissues; that enables to develop advanced bio-based technologies for applications across a wide spectrum of social needs, in order to perform breakthrough in diagnosis, treatment and prevention of diseases. Definitively, Biomechanics is a multi-disciplinary framework towards the enhancing of the environmental health. And this novel perspective does not overcome the classical concept of mechanics of life, but it improves and completes the ancient challenge aiming at understanding mechanisms that regulate living matter, intertwining with the growing advances in science and technology, as well as with the growing high-quality life expectancy.

In this light, advances in computing technology, numerical methods, and modeling techniques able to account for multi-field couplings and multi-physics descriptions - which are typical features in biomechanical problems - have to be continuously pursued, in order to built up suitable modern tools for facing the renewed challenges launched by Biomechanics.

As a matter of fact, enhanced modeling formulations and advanced computational methods open towards the possibility of developing effective computer-aided simulation tools, allowing reliable, predictive and patientspecific biomechanical analyses. Moreover, they may enable to contribute to improving current clinical and diagnostic approaches in a groundbreaking way. For instance, patient-specific treatments might be conceived and adapted according to physiopathological responses of body systems; effects of histological and biochemical rearrangement on tissue constituents might be investigated; and non-invasive techniques for estimating, in an inverselike scheme, the values of histo-mechano-chemical features might be provided.

In this context, modeling and simulation of soft tissues can be considered as one of the open and key research issues $[3,4]$. In this case, biological constructs can undergo large displacement and/or large strains. Moreover, they are characterized by a complex non-linear mechanical behavior, strongly depending on tissue histological features and biological environment. Thus, there is a great need for the development of accurate constitutive models accounting for coupled mechanisms, usually occurring at very different length scales, and involving different physics.

Soft tissues are throughout the whole human body and they include tendons, ligaments, skin, fibrous tissues, muscles, blood vessels. They link, support, and are part of other bio-structures and organs, playing a key role in the biomechanics of many body systems (e.g., musculoskeletal, respiratory, cardiovascular) [5]. Soft tissues are generally fibrous connective tissues which can be either dense or loose, depending on the collagen amount. They consist primarily of elastin, amorphous ground substance, cells and collagen fibers [6].

As confirmed by well-established studies [7], the highly nonlinear constitutive response of soft tissues at the macroscale is strictly related to the hierarchical organization of collagen from nano up to the microscale.

In biological soft tissues, tropocollagen molecules (mainly of type I) can be regarded as one-dimensional structures about $300 \mathrm{~nm}$ long and $1-2 \mathrm{~nm}$ in diameter, made up of three polypeptide strands, each one being a lefthanded helix. The three helices are twisted together into a triple helix (namely, a super helix), representing a cooperative quaternary structure stabilized by covalent cross-links [5]. A single tropocollagen subunit self-assembles in the extracellular matrix with four other collagen molecules to form units that, in turn, assemble themselves into even larger arrays, called fibrils. A collagen fibril, characterized by a diameter between 50 to $500 \mathrm{~nm}$, can be thought as a mesoscale structure between molecule at the nanoscale and fiber at the microscale. In turn, collagen fibrils self-assemble in densely packed tilted bundles, namely collagen fibers, stabilized by lateral proteoglycans [8]. Within this rigidly organized biostructure, molecules interact with each other by means of both inter-molecular covalent cross-links (each of them connecting two molecules) and weak bonds (including hydrogen bonds and other electromagnetic weak interactions), the former being dominant with reference to the fibril's elastic behavior [9].

Collagen fibers in soft tissues can be arranged in 
agreement with a regular (e.g., tendons) or an irregular (e.g., skin) pattern, and regular tissues (that is, with an almost regular fiber arrangement) can be conveniently classified as uni- (e.g., tendons and ligaments) or multi(e.g., arterial walls) directional tissues. Accordingly, different collagen patterns and amounts induce different mechanical responses, namely induce different stiffness and strength features at the macroscale. As a result, specific values of compliance for arterial walls in cardiovascular system, of stiffness for pulmonary tissues in respiratory system, and of extensibility for tendons in musculoskeletal system, are experienced. Moreover, altered tissue response in disease (e.g., aneurism, keratoconus, arthofibrosis) arises from pathological tissue remodeling and arrangement alterations at the nano and microscale, inducing unphysiological histology and biochemical composition. Typical ageing/disease-related mechanical dysfunctions and disorders, such as tissue hyper-extensibility or weakness, that can produce aneurysm pathologies in arterial tissues, can be associated with alterations at different scales [10-16]: in content of tissue constituents, in shape of collagen fibers, in collagen genetic pattern, in density of intermolecular cross-links.

Nevertheless, available non-invasive techniques do not allow to measure directly a number of important histological, mechanical, and biochemical properties of collagenous tissues, such as, for instance but not exclusively, collagen content and fiber waviness, collagen cross-linking, elastin amount, and stiffness of elastin networks.

In this scenario, the development of theoretical results and computational methods for effectively correlating mechano-regulated physio-pathological processes occurring at very different length scales, as well as to identify relationships among alterations and diseases, represents an open challenge at the cutting edge of modern biomechanics.

To date, current modeling approaches widely employed within advanced computational frameworks allow to incorporate only some (but not all) histological data, and include some biochemical effects only through phenomenological laws [17-19]. These models are usually based on parameters having no direct physical or morphological meaning, that have to be suitably tuned in order to reproduce experimental behavior observed at the macroscale. Other approaches, namely structural approaches, aim to link model parameters with tissue arrangement and properties at lower scales, either by micromacro homogenization techniques or by assuming a suitable hyper-elastic macroscopic behavior on the basis of specific tissue microscale features. Effects related to collagen fibers are generally treated as linearly elastic in the first case [20-22], whereas geometric nonlinearities (mainly related to the fiber crimp) and material nonlinearities (induced by nanoscale mechanisms within and among collagen molecules) are often taken into account in hyper-elastic models by choosing a suitable representation of the fiber strain-energy density as in a phenomenological approach $[18,23,24]$. Accordingly, some microscale features (such as the crimp shape or the thickness of collagen fibers) and any direct relationship with the molecular scale are usually neglected, despite their well-established physio-pathological relevance [10-16].

On the other hand, if tissue modeling aims not only to reproduce the mechanical response at the macroscale but also, in a patient-specific framework, to contribute for diagnostic purposes, as well as to describe the tissue response evolution induced by physio-pathological alterations in tissue arrangement, each model parameter should be directly associated with a well-recognizable and measurable property.

This requirement can be satisfied if the tissue structured hierarchical arrangement is explicitly described, possibly reducing model complexity by means of multiscale homogenization techniques. Such an approach, employed for example in $[25,26]$, is referred to as a structural multiscale method. It consists in regarding the tissue at the macroscale as a fiber-reinforced composite material, wherein properties of reinforcement phase are recovered by mechanical models at smaller (than the macro) length scales, coupled each other by means of consistent inter-scale relationships. Accordingly, the equivalent responses of tissue substructures at different scales can be analytically derived and consistently integrated and upscaled, allowing to include at the macroscale the dominant mechanisms occurring at smaller scales. In some way, the structural multiscale approach exploits the rationale followed by nature in designing tissues and building organs.

Structural multiscale elastic constitutive models for collagen-rich tissues have been recently proposed [3, 26-30], based on homogenization techniques that explicitly incorporate nanoscale and microscale mechanisms, as well as their coupling effects. By describing histological alterations at nano, micro and macro scales, those models have been proved to be able to give useful indications on the deep link between histology and mechanical response of both collagenous tissues and body structures [28-30]. This approach can be generalized, in the same modeling framework, for including also damage evolution and inelastic effects at different scales, generally induced by both mechanical and non-mechanical sources $[31,32]$.

In order to account for nano and microscale effects in macroscale tissue response, a key aspect is represented by the modeling of collagen behavior at molecular (nanoscale) and fibril/fiber (microscale) levels, and by the consistent coupling of these single-scale descriptions.

Nanomechanical response of collagen molecules is 
governed by both entropic thermal fluctuation and energetic stretching mechanisms [7,26,33,34], usually addressed via numerical atomistic computations [35]. Recently, an effective modeling approach, simply integrable in a continuum framework that addresses the equivalent mechanical response of the tissue at the macroscale, has been proposed $[3,4,29,30]$. It is based on the assumption that a collagen molecule can be described by an equivalent zero-dimensional elastic nano-structure, wherein entropic and energetic mechanisms act as in series.

Therefore, referring to soft living tissues, structural multiscale models reveal as really promising for developing virtual simulation tools, that are efficient from a computational point of view and are able, at the same time, to account for patient-specific features, not only in geometric description of the tissue domains, but also for the accurate representation of the tissue mechanical properties. As a result, the effects of changes in histological arrangement or biochemical environment on the overall macroscopic functionality of tissues and organs could be predicted. Thereby, really customized pharmacological treatments and therapeutic strategies could be conveniently designed and applied. Moreover, structural multiscale methods based on consistent homogenization procedures can be successfully integrated with numerical submodeling techniques, allowing to analyze stress and strain localization at the microscale, around and within cells [4,29]. Accordingly, clear quantitative indications on cellular response to macroscale mechanical stimuli can be furnished, contributing to understanding activation and evolution mechanisms of mechano-regulated biological processes, and opening also to the possibility to quantify critical states related to biological transitions towards pathological conditions. Finally, parametric biomechanical simulations of tissues and organs based on a multiscale structural framework might be coupled with non-invasive in-vivo histological and functional measures. Thereby, following an inverse-like scheme, indirect estimates of histo-mechano-chemical features, otherwise unknown, could be furnished by aiming to improve diagnostic procedures.

\section{REFERENCES}

[1] Sanan, A. and Rengachary, S.S. (1996) The history of spinal biomechanics. Neurosurgery, 39, 657-68. http://dx.doi.org/10.1097/00006123-199610000-00001

[2] Porter, R. (1997) The greatest benefit to mankind: A medical history of humanity from antiquity to the present. Harper Collins, New York.

[3] Marino, M. and Vairo, G. (2013) Multiscale elastic models of collagen bio-structures: From cross-linked molecules to soft tissues. In: Gefen, A., Ed., Multiscale Computer Modeling in Biomechanics and Biomedical Engi- neering. Springer, Berlin-Heidelberg. Studies in Mechanobiology, Tissue Engineering and Biomaterials, 14, 73102. http://dx.doi.org/10.1007/8415 $2012 \quad 154$

[4] Marino, M. and Vairo, G. (2013) Computational modelling of soft tissues and ligaments. In: Jin, Z., Ed., Computational Modelling of Biomechanics and Biotribology in the Musculoskeletal System: Biomaterials and Tissues, Woodhead Publishing Series in Biomaterials, 81, Woodhead Publishing Limited, Cambridge.

[5] van Holde, K.E. and Matthews, C. (1995) Biochemistry. Publishing Company Inc., Benjamin/Cummings.

[6] Martini, F.H., Timmons, M.J. and Tallitsch, R.B. (1994) Human anatomy. Prentice Hall, Upper Saddle River.

[7] Fratzl, P. (2008) Collagen: Structure and mechanics. Springer-Verlag, New York. http://dx.doi.org/10.1007/978-0-387-73906-9

[8] Pins, G.D., Christiansen, D.L., Patel, R. and Silver, F.H. (1997) Self-assembly of collagen fibers. Influence of fibrillar alignment and decorin on mechanical properties. Biophysical Journal, 73, 2164-2172. http://dx.doi.org/10.1016/S0006-3495(97)78247-X

[9] Eyre, D.R., Weis, M.A. and Wu, J.J. (2008) Advances in collagen cross-link analysis. Methods, 45, 65-74. http://dx.doi.org/10.1016/j.ymeth.2008.01.002

[10] Bruel, A. and Oxlund, H. (1996) Changes in biomechanical properties, composition of collagen and elastin, and advanced glycation endproducts of the rat aorta in relation to age. Atherosclerosis, 127, 155-165 http://dx.doi.org/10.1016/S0021-9150(96)05947-3

[11] Bruel, A., Ørtoft, G. and Oxlund, H. (1998) Inhibition of cross-links in collagen is associated with reduced stiffness of the aorta in young rats. Atherosclerosis, 140, 135145. http://dx.doi.org/10.1016/S0021-9150(98)00130-0

[12] Mao, J.R. and Bristow, J. (2001) The Ehlers-Danlos syndrome: On beyond collagens. Journal of Clinical Investigation, 107, 1063-1069. http://dx.doi.org/10.1172/JCI12881

[13] Bailey, A.J. (2001) Molecular mechanisms of ageing in connective tissues. Mechanisms of Ageing and Development, 122, 735-755. http://dx.doi.org/10.1016/S0047-6374(01)00225-1

[14] Carmo, M., Colombo, L., Bruno, A., Corsi, F.R.M., Roncoroni, L., Cuttin, M.S., Radice, F., Mussini and E., Settembrini, P.G. (2002) Alteration of Elastin, Collagen and their cross-links in abdominal aortic aneurysms. European Journal of Vascular and Endovascular Surgery, 23, 543-549. http://dx.doi.org/10.1053/ejvs.2002.1620

[15] Järvinen, T.A.H., Järvinen, T.L.N., Kannus, P., Jòzsa, L. and Järvinen, M. (2004) Collagen fibres of the spontaneously ruptured human tendons display decreased thickness and crimp angle. Journal of Orthopaedic Research, 22, 1303-1309. http://dx.doi.org/10.1016/j.orthres.2004.04.003

[16] Couppé, C., Hansen, P., Kongsgaard, M., Kovanen, V., Suetta, C., Aagaard, P., Kjær, M. and Magnusson, S.P. (2009) Mechanical properties and collagen cross-linking of the patellar tendon in old and young men. Journal of Applied Physiology, 107, 880-886. 
http://dx.doi.org/10.1152/japplphysiol.00291.2009

[17] Balzani, D., Neff, P., Schröder, J. and Holzapfel, G.A. (2006) A polyconvex framework for soft biological tissues. Adjustment to experimental data. International Journal of Solids and Structures, 43, 6052-6070. http://dx.doi.org/10.1016/j.ijsolstr.2005.07.048

[18] Holzapfel, G.A., Gasser, T.C. and Ogden, R.W. (2000) A new constitutive framework for arterial wall mechanics and a comparative study of material models. Journal of Elasticity, 61, 1-48. http://dx.doi.org/10.1023/A:1010835316564

[19] Holzapfel, G. and Gasser, T.C. (2001) A viscoelastic model for fiber-reinforced composites at finite strains: Continuum basis, computational aspects and applications. Computer Methods in Applied Mechanics and Engineering, 190, 4379-4403. http://dx.doi.org/10.1016/S0045-7825(00)00323-6

[20] Comninou, M. and Yannas, I.V. (1976) Dependance of stress-strain nonlinearity of connective tissues on the geometry of collagen fibers. Journal of Biomechanics, 9, 427-433. http://dx.doi.org/10.1016/0021-9290(76)90084-1

[21] Lanir, Y. (1979) A structural theory for the homogeneous biaxial stress-strain relationships in flat collagenous tissues. Journal of Biomechanics, 12, 423-436. http://dx.doi.org/10.1016/0021-9290(79)90027-7

[22] Freed, A.D. and Doehring, T.C. (2005) Elastic model for crimped collagen fibrils. Journal of Biomechanical Engineering-ASME, 127, 587-593. http://dx.doi.org/10.1115/1.1934145

[23] Holzapfel, G.A., Gasser, T.C. and Stadler, M. (2002) A structural model for the viscoelastic behavior of arterial walls: Continuum formulation and finite element analysis. European Journal of Mechanics-A/Solids, 21, 441-463. http://dx.doi.org/10.1016/S0997-7538(01)01206-2

[24] Ciarletta, P., Micera, S., Accoto, D. and Dario, P. (2006) A novel microstructural approach in tendon viscoelastic modelling at the fibrillar level. Journal of Biomechanics, 39, 2034-2042. http://dx.doi.org/10.1016/i.jbiomech.2005.06.025

[25] Tang, H., Buehler, M.J. and Moran, B. (2009) A constitutive model of soft tissue: from nanoscale collagen to tissue continuum. Annals of Biomedical Engineering, 37, 1117-1130. http://dx.doi.org/10.1007/s10439-009-9679-0

[26] Maceri, F., Marino, M. and Vairo, G. (2010) A unified multiscale mechanical model for soft collagenous tissues with regular fiber arrangement. Journal of Biomechanics,
43, 355-363. http://dx.doi.org/10.1016/j.jbiomech.2009.07.040

[27] Maceri, F., Marino, M. and Vairo, G. (2010) From crosslinked collagen molecules to arterial tissue: A nano-micro-macroscale elastic model, Acta Mechanica Solida Sinica, 23, 98-108.

[28] Maceri, F., Marino, M. and Vairo, G. (2011) An insight on multiscale tendon modelling in muscle-tendon integrated behavior. Biomechanics and Modeling in Mechanobiology, 11, 505-517.

http://dx.doi.org/10.1007/s10237-011-0329-8

[29] Marino, M. and Vairo, G. (2012) Stress and strain localization in stretched collagenous tissues via a multiscale modeling approach. Computer Methods in Biomechanics and Biomedical Engineering.

http://dx.doi.org/10.1080/10255842.2012.658043

[30] Maceri, F., Marino, M. and Vairo, G. (2013) Age-dependent arterial mechanics via a multiscale elastic approach. International Journal for Computational Methods in Engineering Science and Mechanics, 14, 141-151. http://dx.doi.org/10.1080/15502287.2012.744114

[31] Maceri, F., Marino, M. and Vairo, G. (2012) Elastodamage modelling of biopolymer molecules response. Computer Modeling in Engineering and Sciences, 87, 461-482. http://dx.doi.org/10.3970/cmes.2012.087.461

[32] Balzani, D., Brinkhues, S. and Holzapfel, G.A. (2012) Constitutive framework for the modeling of damage in collagenous soft tissues with application to arterial walls. Computer Methods in Applied Mechanics and Engineering, 213, 139-151. http://dx.doi.org/10.1016/j.cma.2011.11.015

[33] Bozec, L. and Horton, M. (2005) Topography and mechanical properties of single molecules of type I collagen using atomic force microscopy. Biophysical Journal, 88, 4223-4231.

http://dx.doi.org/10.1529/biophysj.104.055228

[34] Buehler, M.J. and Wong, S.Y. (2007) Entropic elasticity controls nanomechanics of single tropocollagen molecules. Biophysical Journal, 93, 37-43.

http://dx.doi.org/10.1529/biophysj.106.102616

[35] Buehler, M.J. (2008) Nanomechanics of collagen fibrils under varying cross-link densities: Atomistic and continuum studies. Journal of the Mechanical Behavior of Biomedical Materials, 1, 59-67.

http://dx.doi.org/10.1016/j.jmbbm.2007.04.001 